\title{
A theoretical analysis of the free axial vibration of non-local rods with fractional continuum mechanics
}

\author{
W. Sumelka $(\mathbb{D} \cdot$ R. Zaera $\cdot$ J. Fernández-Sáez
}

Received: 21 May 2014/Accepted: 17 March 2015/Published online: 31 March 2015

(C) The Author(s) 2015. This article is published with open access at Springerlink.com

\begin{abstract}
In this paper we present a theoretical analysis of free axial vibrations of rods described in terms of the fractional continuum mechanics. This formulation is able to capture the size effect arising in different problems of solid mechanics. The natural frequencies and modal shapes for clamped rods are obtained and the effects of the derivative order, as well as the corresponding length-scale parameters in the fractional model are discussed. The results are compared with those derived from the Eringen non-local elasticity theory, which is an approach widely used to model structures showing size effects.
\end{abstract}

Keywords Non-local models $\cdot$ Rods $\cdot$ Fractional calculus

\footnotetext{
W. Sumelka ( $\square)$

Institute of Structural Engineering, Poznań University of Technology, Piotrowo 5 street, 60-969 Poznań, Poland e-mail: wojciech.sumelka@put.poznan.pl

\section{R. Zaera · J. Fernández-Sáez}

Department of Continuum Mechanics and Structural

Analysis, University Carlos III of Madrid,

28911 Leganés, Madrid, Spain

e-mail: ramon.zaera@uc3m.es

J. Fernández-Sáez

e-mail: ppfer@ing.uc3m.es
}

\section{Introduction}

The formalism based on the classical Continuum Mechanics has permitted to develop powerful and reliable simulation tools to solve complex problems in the field of structural and solid mechanics. However, the matter is discrete and heterogeneous, and the hypotheses of the classical continuum are no longer valid for nanostructures used as NEMS, nano-machines or biosensors $[6,14,20,27,50,72]$, granular materials [29] or when the size of the solid-or the wavelength of the loading excitation-is comparable to that of its microstructure. It is well known that classical continuum mechanics (CCM) is size-independent. Because of the structure of its governing equations, it lacks an internal length and, for this reason, it cannot predict any size effect and may fail when effects like size-dependency and scaling of mechanical phenomena play a crucial role.

The above problems could be addressed using discrete models but all of them require a great computational effort. This fact provides a motivation towards developing generalized continuum mechanics theories that are able to capture the size effects by introducing intrinsic lengths in its formulation.

Within this category fall the classical couple stress theory and the strain gradient theory, started in 1960s with the works of Mindlin and Tiersten [54], Kröner [37], Toupin [84, 85], Green and Rivlin [26], Mindlin [51, 52] and Mindlin and Eshel [53]. Nevertheless, these theories are excessively complex with too many 
parameters and equations. Due to the difficulties in determining microstructure-dependent parameters $[39,48,95]$ models involving a reduced number of constants, like the modified couple stress theory [66, 67, 94], are desirable.

A size-dependent continuum theory which contains only one material length scale parameter is the nonlocal continuum mechanics initiated by Eringen and coworkers [15-17], which has has been widely used to analyse many problems, such as wave propagation, dislocation, and crack singularities.

Peddieson et al. [68] used this theory for the first time to solve problems involving nanostructures. Subsequently, the Eringen non-local theory of elasticity has been used to address the behavior of nanobeams [32, 44, 46, 71, 74, 89, 90, 93], nanobeams under rotation $[3,57,64,70]$, nanorods $[34,42,45,56$, $59,61,62,81]$, nanoplates [30, 33, 58], cylindrical nanoshells [31, 91, 92], conical nanoshells [21, 43, 86], nanorings [55, 88], spherical nanoshells [24, 87, 96], as well as particles [25] and carbon nanotubes (CNTS) [2, 10, 19, 22, 28, 60, 63, 75, 97].

A different approach to non-local mechanics has been recently introduced in the context of fractional calculus. Fractional calculus (FC) is a branch of mathematical analysis that studies the differential operators of an arbitrary (real or complex) order [35, 69]. The attractiveness of FC application lays in the fact that: (1) fractional differential operators are nonlocal, and (2) there are many definitions of fractional derivatives. In the last decades fractional differential equations have been used to capture physical phenomena that cannot be caught by classical differential models. In the frame of Solid Mechanics this mathematical approach has been introduced especially in describing viscous behavior of materials, where the non-locality is taken with respect to the time variable (memory) (cf. [47] and cites therein). However it has been also used in the stress space through the fractional gradient of plastic potential in order to control magnitude and direction of viscoplastic strain evolution [79], or in the space variable. In the latter case, the order of the derivative is usually lower than that of the classical one, a major asset in comparison to gradient theories which provide derivatives of the displacement of higher orders thus needing extra boundary conditions to be solved, whose physical meaning is still unclear [9].
Seminal works of spatial fractional calculus applied to one-dimensional media may be found in the past decade. The idea to include a fractional term in the governing equation of the elastic problem has been proposed by Lazopoulos [40]. He starts by assuming a strain energy consisting of a local part of the kind $E \varepsilon^{2} / 2, E$ being the Young modulus and $\varepsilon$ the longitudinal strain, and a non-local part with fractional derivatives. Variation of the total strain energy functional with respect to $\varepsilon$ yields, under the assumption of vanishing boundary conditions, to a stress-strain relation involving Riemman-Liouville fractional integrals. Di Paola and Zingales [12] showed that this approach is equivalent to the strong non-local theory of long-range forces $[18,38]$ if a specific kernel function is adopted for the convolution integral. To overcome inconsistencies that may appear at free boundaries, these authors proposed two different-but totally equivalent-mechanical models in which long-range forces in the stress-strain relation are represented by a Marchaud fractional derivative for both unbounded and bounded domains [9, 12]. All these works focus on static behavior, however fractional calculus has been also considered to model the dynamic behavior of 1D non-local media. Cottone et al. [11] used the previous models for the analysis of dispersive effects in 1D elastic waveguides. Likewise, Drapaca and Sivaloganathan [13], developed the foundations for a non-local model of continuum mechanics and applied their framework to the propagation of longitudinal waves in a thin finite bar. Sapora et al. [73] investigated the wave propagation using spatial derivatives of non-integer order greater than one. Other fractional approach is the one proposed by Tarasov [82], in which elastic continuum is interpreted as the limit of discrete systems with long range interactions. The transition between integral and gradient non-local elasticity in terms of fractional operators comes out naturally [83]. Finally, Carpinteri et al. [8] recently presented a theoretical analysis of the mechanical behaviour of one-dimensional structure made of a material whose constitutive law provides the stress as the fractional integral of the strain field. This fractional non-local model was proved to be the continuum limit of a discrete lattice with three levels of long-range interactions, thus being able to capture the interactions between bulk and surface material points. 
The aim of this paper is to present a detailed theoretical study of free axial vibration of clamped rods using the fractional continuum mechanics (FCM) approach proposed by Sumelka [78, 80], in which the kinematics is developed in a finite deformation frame-in contrast to previous works where small deformations are only considered-and different intermediate fractional configurations arise for different values of the derivative order. This formulation offers a clear analogy between the generalised fractional measures of the deformation, such as fractional deformation gradients or fractional strains, and their counterparts in the classical continuum mechanics. Therefore, the interpretation and dimensions of fractional strains remain unchanged with respect to the classical ones. In this sense, there is no need to introduce non-standard definitions for material properties [4, 7]. Moreover, the domain over which the non-local effects are taken into account can be selected, thus it may be a sub-domain of the whole solid. This way, the definition of the length scale is more accurate. It is important to emphasise that thorough this paper we utilise small fractional deformation case, but resulting from finite fractional deformations concept [78, 80]. The structure of balance equations remains unchanged (such result is similar to that presented in [4]). Other relevant differences with previous approaches $[4,7,13,36]$ will be clarified in Sect. 2.1.2. The natural frequencies and modal shapes are obtained and the effects of the derivation order and length-scale parameter are discussed and contrasted with those obtained with the solutions derived from the classical local model and the non-local Eringen model [5]. The model provides a basis for the study of the dynamic behaviour of onedimensional elastic structures showing scale effects, in the framework of the proposed FCM formulation.

\section{Problem formulation}

\subsection{Fractional model}

\subsubsection{General comments}

The current formulation introduces non-locality in spatial variable and utilises Riesz-Caputo (RC) fractional derivative $[1,23]$. In the following section the fundamental concepts governing the RC fractional continua are presented to give the insight into the overall concept of CCM generalisation by FC application-for a more detailed discussion see [77, 78, 80].

As mentioned, the presented concept of the RC fractional continua differs from the classical continua by replacing the classical deformation gradient (first derivative of motion) with the fractional deformation gradient (fractional derivative of motion) [76, 77, 80]. Therefore, the fractional deformation gradient operates by analogy to classical deformation gradient. The important difference is that fractional deformation gradient is non-local; thus all other related measures (e.g. strain tensor) are also non-local. In this sense, deformation measurements are given by fractional deformation gradient mapping.

As it will be observed, the presented FCM formulation introduces three important phenomenological concepts: (1) the type of fractional derivative; (2) the order of fractional differentiation $\alpha$; and (3) the size of nonlocal surrounding $\ell_{f}$. They all are important and should be thought as material parameters i.e. they control the amount of information which should be taken into account (parameter $\ell_{f}$ ), and the way in which this information influences the point of interest (type of fractional derivative and parameter $\alpha$ ). It is noticeable that such results, are analogous to the model by Drapaca and Sivaloganathan [13] where there are also two parameters which control the non-locality, namely: one connected with space, and the second (order of derivative) interpreted as a measure of the dynamic deformation possibly due to e.g. electro-magnetic, thermic and/or chemical processes. Thus, using adequate non-local phenomenological parameters, it is possible to describe in homogenised sense, real heterogeneous material structure, intrinsic processes, and capture the size effect i.e. the behaviour of such material depends on the size of the specimen/structure.

\subsubsection{RC fractional continua-small deformations case}

The description is given in the Euclidean space. We refer to $\mathcal{B}$ as the reference configuration of the continuum body while $\mathcal{S}$ denotes its current configuration. Points in $\mathcal{B}$ are denoted by $\mathbf{X}$ and in $\mathcal{S}$ by $\mathbf{x}$. The coordinate system for $\mathcal{B}$ is denoted by $\left\{X_{A}\right\}$ with base $\mathbf{E}_{A}$ and for $\mathcal{S}$ we have $\left\{x_{a}\right\}$ with base $\mathbf{e}_{a}$. 
We generalise the classical deformation gradient and its inverse as follows

$$
\begin{aligned}
& \underset{X}{\tilde{\mathbf{F}}}(\mathbf{X}, t)=\ell_{X}^{\alpha-1} D_{X}^{\alpha} \phi(\mathbf{X}, t), \quad \text { or } \\
& \underset{X_{a A}}{\tilde{F}}=\ell_{A}^{\alpha-1}{ }_{X_{A}}^{\alpha} \phi_{a} \mathbf{e}_{a} \otimes \mathbf{E}_{A},
\end{aligned}
$$

and

$$
\begin{aligned}
& \underset{x}{\tilde{\mathbf{F}}}(\mathbf{x}, t)=\ell_{x}^{\alpha-1} D_{x}^{\alpha} \varphi(\mathbf{x}, t), \quad \text { or } \\
& \tilde{F}_{A a}=\ell_{a}^{\alpha-1} D_{x_{a}}^{\alpha} \varphi_{A} \mathbf{E}_{A} \otimes \mathbf{e}_{a},
\end{aligned}
$$

where $\underset{X}{\tilde{\mathbf{F}}}$ and $\underset{x}{\tilde{\mathbf{F}}}$ are fractional deformation gradients, $\ell_{X}$ and $\ell_{x}$ are length scales in $\mathcal{B}$ and $\mathcal{S}$, respectively, $\phi$ defines the regular motion of the material body while $\varphi$ defines its inverse [49]. We assume additionally that $\ell_{X}=\ell_{x}=\ell_{f}$. The fractional differential operator $D^{\alpha}$ applied to a function $f(\chi)(\chi \in(a, b) \subseteq \mathbb{R})$ is defined as the $\mathrm{RC}$ fractional derivative:

$$
\begin{aligned}
{ }_{a}^{R C} D_{b}^{\alpha} f(\chi)= & \frac{1}{2} \frac{\Gamma(2-\alpha)}{\Gamma(2)} \\
& \left({ }_{a}^{C} D_{\chi}^{\alpha} f(\chi)+(-1){ }_{\chi}^{n C} D_{b}^{\alpha} f(\chi)\right),
\end{aligned}
$$

where $\alpha>0$ denotes the real order of the derivative, $D$ denotes 'derivative' (RC stands for Riesz-Caputo), $a, \chi, b$ are so called terminals, $n=\lfloor\alpha\rfloor+1,\lfloor\bullet\rfloor$ is the floor function, and the factor $\frac{\Gamma(2-\alpha)}{\Gamma(2)}$ appears for objectivity reasons, i.e. when the superimposed rigid-body motion on the current configuration is considered (cf. [80]). The terminals $a$ and $b$ are in the relation between with the physical length scale of a particular material. In Eq. (3) ${ }_{a}^{C} D_{\chi}^{\alpha} f(\chi),{ }_{\chi}^{C} D_{b}^{\alpha} f(\chi)$ are respectively the left and right Caputo's fractional derivatives [35, 41, 69],

$$
\begin{aligned}
& { }_{a}^{C} D_{\chi}^{\alpha} f(\chi)=\frac{1}{\Gamma(n-\alpha)} \int_{a}^{\chi} \frac{f^{(n)}(\tau)}{(\chi-\tau)^{(\alpha-n+1)}} d \tau, \\
& { }_{\chi}^{C} D_{b}^{\alpha} f(\chi)=\frac{(-1)^{n}}{\Gamma(n-\alpha)} \int_{\chi}^{b} \frac{f^{(n)}(\tau)}{(\chi-\tau)^{(\alpha-n+1)}} d \tau,
\end{aligned}
$$

$f^{(n)}$ being the $n$-th derivative of function $f$. When $\alpha$ is an integer, the usual definition of derivative is recovered [1,23]. In the current work, values of $\alpha$ in the range $(0,1]$ will be considered.

It is worth to notice that the introduction of fractional deformation gradients results that one can think that deformation is observed in 'fractional' space. It means that we operate on fractional spatial and material line, surface and volume elements. As previously mentioned, in a later step one can redefine all well known measures of deformations like Green-Lagrange or Euler-Almansi strain tensors, as well as left/right Cauchy-Green tensors based on the proposed fractional deformation gradients; similarly as in CCM, in FCM one can define the strain measures in terms of displacements.

Applying small deformation assumption, understood as omitting higher order terms, we obtain the infinitesimal fractional Cauchy strain tensor

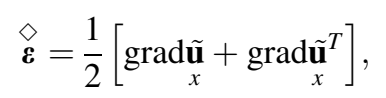

where $\stackrel{\diamond}{\boldsymbol{\varepsilon}}$ stands for infinitesimal fractional Cauchy

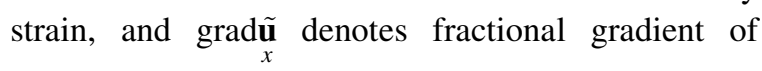
displacement $\mathbf{u}$. It is clear that when applying $\alpha=1$ the classical Cauchy strain tensor is recovered

$$
\stackrel{\diamond}{\boldsymbol{\varepsilon}}=\boldsymbol{\varepsilon}=\frac{1}{2}\left[\operatorname{gradu}+\operatorname{grad} \mathbf{u}^{T}\right] .
$$

For 1D deformation, from Eq. (6) we have (Fig. 1)

$$
{\stackrel{\diamond}{\varepsilon_{x}}}=\ell_{f}^{\alpha-1} \frac{1}{2} \frac{\Gamma(2-\alpha)}{\Gamma(2)}\left({ }_{x-\ell_{f}}^{C} D_{x}^{\alpha} u-{ }_{x}^{C} D_{x+\ell_{f}}^{\alpha} u\right) \text {. }
$$

where $u$ is the displacement in $x$ direction, and the same distance between terminals has been considered $\left(\ell_{f}=x-a=b-x\right)$. As stated before, when $\alpha=1$

$$
\stackrel{\diamond}{\varepsilon}_{x}=\varepsilon_{x}=\frac{d u}{d x} .
$$

The result given by Eq. (8) allows to observe the differences and similarities with those obtained in $[4$, 7, 36]. Thus, the important differences are: Eq. (8) is obtained as a special case of general fractional finite strains; the fractional derivative operates on the finite interval $[a, b]$; the length scale $\ell_{f}$ is given explicitly, and it is in the relation with the interval over which the fractional differential operator acts; the strain is nondimensional like in a classical case. Finally, assuming that all information from the body influences the point of interest (i.e. in $1 \mathrm{D}$ the interval $[a, b]$ coincides with body boundaries) we observe some similarities. Namely, in this case, from the mathematical point of view the new model coincides with previous one, however the physical units remain different. 
Fig. 1 Non-local fractional strain concept for $1 \mathrm{D}$ case

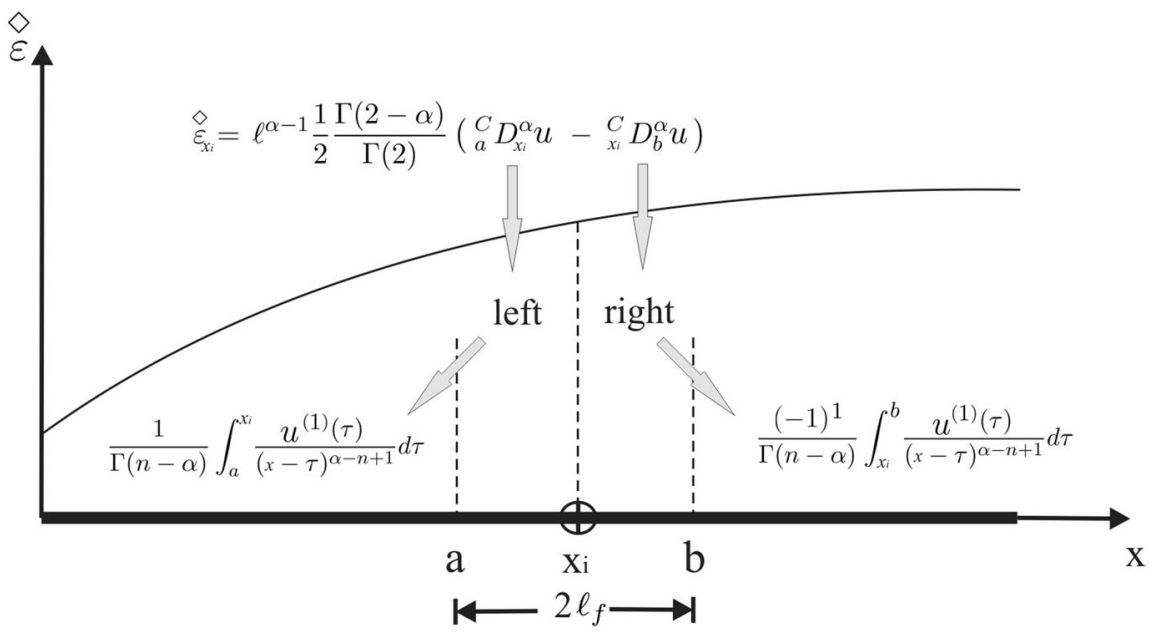

$u(\xi, t)=U(\xi) e^{i \omega_{f} t}$

\subsubsection{Algebraic eigenproblem for $1 D$ case}

We consider a bar of cross section area $\Omega$, length $L$, density $\rho$ and Young modulus $E$, submitted to body force $b_{x}$ per unit length. The problem of 1D linear elasticity under FCM is governed by the following equations

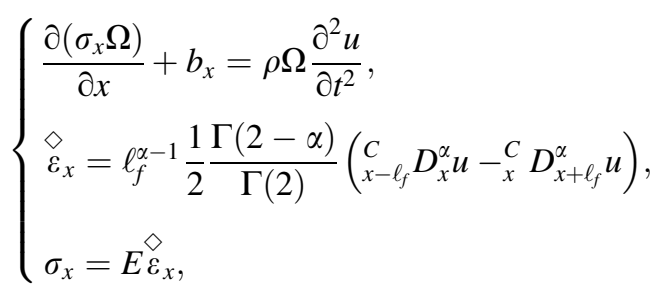

as well as specific initial and boundary conditions. In previous equations, $\sigma_{x}$ is the stress.

Considering constant cross section and $b_{x}=0$, Eq. (10) are reduced to a single partial (fractional) differential equation

$E \ell_{f}^{\alpha-1} \frac{1}{2} \frac{\Gamma(2-\alpha)}{\Gamma(2)} \frac{\partial}{\partial x}\left({ }_{x-\ell_{f}}^{C} D_{x}^{\alpha} u-{ }_{x}^{C} D_{x+\ell_{f}}^{\alpha} u\right)=\rho \frac{\partial^{2} u}{\partial t^{2}}$,

Assuming now a new dimensionless spatial variable $\xi=x / L$ and $\overline{\ell_{f}}=\frac{2 \ell_{f}}{L}$, Eq. (11) reads

$$
\begin{aligned}
& E\left(\overline{\ell_{f}} / 2\right)^{\alpha-1} \frac{1}{L^{2}} \frac{1}{2} \frac{\Gamma(2-\alpha)}{\Gamma(2)} \frac{\partial}{\partial \xi}\left({ }_{\xi-\ell_{f} / 2}^{C} D_{\xi}^{\alpha} u-{ }_{\xi}^{C} D_{\xi+\ell_{f} / 2}^{\alpha} u\right) \\
& \quad=\rho \frac{\partial^{2} u}{\partial t^{2}},
\end{aligned}
$$

For the solution of the previous equation we consider separation of variables $\omega_{f}=\omega_{f}\left(\alpha, \ell_{f}\right)$ being the natural frequency for the non-local fractional model. Then, Eq. (12) leads to the following spatial fractional differential equation

$$
\begin{aligned}
& \left(\overline{\ell_{f}} / 2\right)^{\alpha-1} \frac{1}{2} \frac{\Gamma(2-\alpha)}{\Gamma(2)} \frac{\partial}{\partial \xi}\left({ }_{\xi \xi \overline{\ell_{f}} / 2}^{C} D_{\xi}^{\alpha} U(\xi)-{ }_{\xi}^{C} D_{\xi+\overline{\ell_{f}} / 2}^{\alpha} U(\xi)\right) \\
& +\lambda_{f}^{2} U(\xi)=0 .
\end{aligned}
$$

with $\lambda_{f}^{2}=\omega_{f}^{2} \rho L^{2} / E$. For clamped-clamped boundaries, the conditions $U(0)=U(1)=0$ are imposed to the spatial variable.

Because the analytical solutions of the fractional differential equations are very limited, the freevibration problem governed by Eq. (14) is obtained numerically. Therefore we apply the approximations of left and right Caputo's spatial derivatives according to the expressions proposed in [41, 65]. The domain $\xi \in[0,1]$ is divided in $r$ intervals of equal length (see Fig. 2) and discretization points are identified by the following rule

$$
\xi_{i}=\xi_{0}+i \frac{1}{r}
$$

with $\xi_{0}=0, r \in \mathbb{Z}$ where $\mathbb{Z}$ denotes the set of all integers. Approximations are then given by:

- for the left sided derivatives:

$$
\begin{aligned}
& \bar{a}=\xi_{0}^{\bar{a}}<\xi_{1}^{\bar{a}}<\ldots<\xi_{j}^{\bar{a}}<\ldots<\xi_{p}^{\bar{a}}=\xi \\
& h=\frac{\xi_{p}^{\bar{a}}-\xi_{0}^{\bar{a}}}{p}=\frac{\xi-\bar{a}}{p}, \quad p \geq 2
\end{aligned}
$$


Fig. 2 The discretization of a $1 \mathrm{D}$ body-dimensionless case

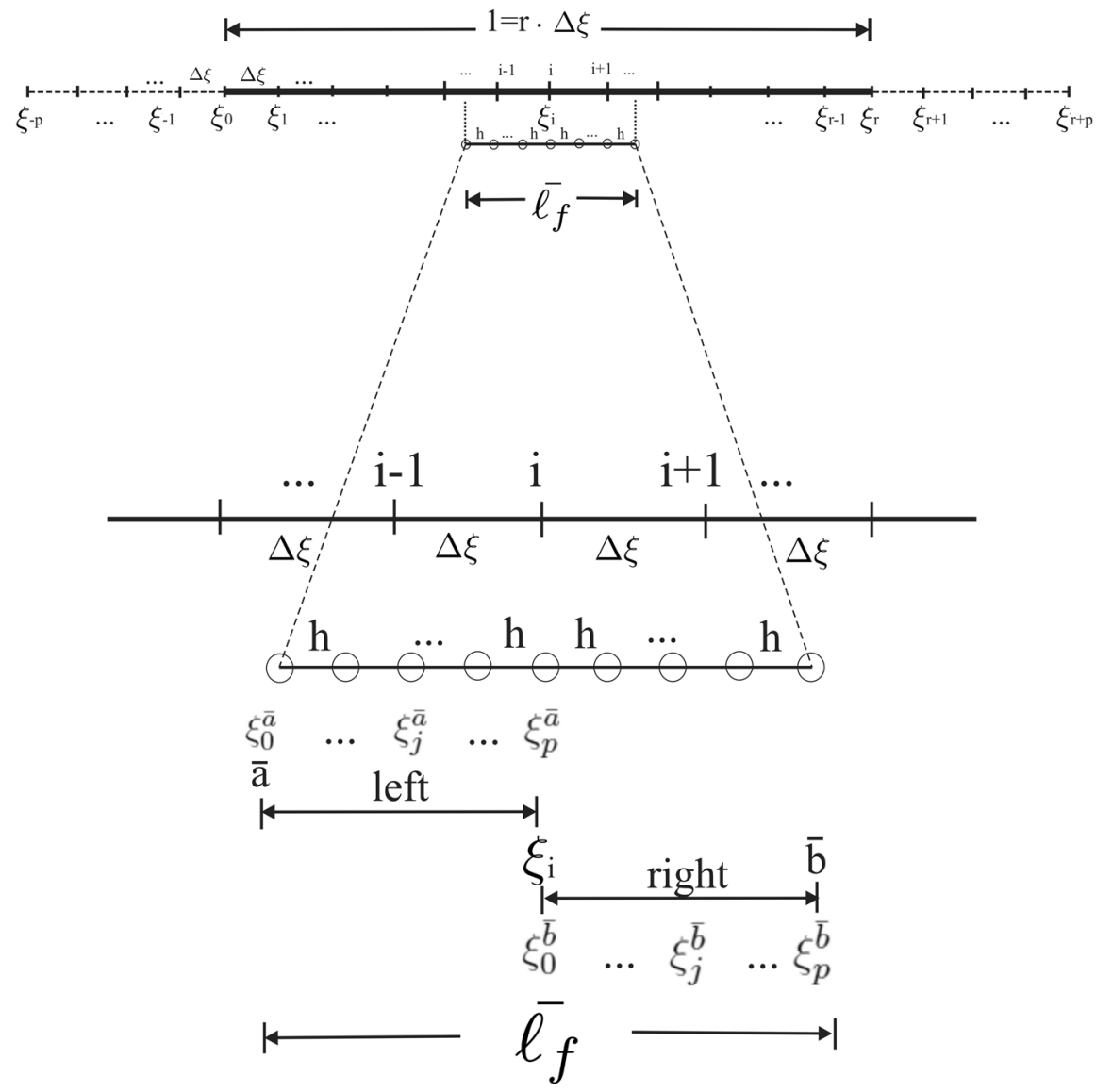

$$
\begin{aligned}
& \xi_{j}^{\bar{b}}=\xi_{0}^{\bar{b}}+j h \\
& \left.{ }_{\xi}^{C} D_{\bar{b}}^{\alpha} U(\xi)\right|_{\xi=\xi_{0}^{\bar{b}}} \cong(-1)^{n} \frac{h^{n-\alpha}}{\Gamma(n-\alpha+2)} \\
& \left\{\left[(p-1)^{n-\alpha+1}-(p-n+\alpha-1) p^{n-\alpha}\right] U^{(n)}\left(\xi_{p}^{\bar{b}}\right)\right. \\
& \quad+U^{(n)}\left(\xi_{0}^{\bar{b}}\right)+\sum_{j=1}^{p-1}\left[(j+1)^{n-\alpha+1}-2 j^{n-\alpha+1}\right. \\
& \left.\left.\quad+(j-1)^{n-\alpha+1}\right] U^{(n)}\left(\xi_{j}^{\bar{b}}\right)\right\},
\end{aligned}
$$

where $\bar{b}=b / L$.

Close to the boundaries, approximation of fractional derivatives may be calculated including additional virtual points, as shown in Fig. 2 (see [80] for details).

Finally we obtain the following algebraic eigenproblem 


$$
\left(\boldsymbol{K}+\lambda_{f}^{2} \boldsymbol{I}\right) \boldsymbol{U}_{0}=0,
$$

where $\boldsymbol{K}$ is the fractional stiffness matrix, $\boldsymbol{I}$ is the identity matrix, and $\boldsymbol{U}_{0}$ is a vector whose elements are the displacements at discretization points (eigenmode). The elements in $\boldsymbol{K}$ depend on selected value of $p$ in the approximations given by Eqs. (18) and (21). Assuming $\alpha \in(0,1) \rightarrow n=1$, the $i$-th row in Eq. (22), describing the behaviour of $i$-th node, reads (cf. Fig. 2):

$$
\begin{aligned}
& \mathrm{A}\left[\mathrm{B} U_{0}^{\bar{a}^{\prime \prime}}+\mathrm{C}^{\bar{a}}(j) U_{j}^{\bar{a}^{\prime \prime}}+U_{i}+\mathrm{C}^{\bar{b}}(j) U_{j}^{\bar{b}^{\prime \prime}}+\mathrm{B} U_{p}^{\bar{b}^{\prime \prime}}\right] \\
& +\lambda_{f}^{2} U_{i}=0,
\end{aligned}
$$

where

$$
\begin{aligned}
& \mathrm{A}=\left(\frac{\overline{\ell_{f}}}{2}\right)^{\alpha-1} \frac{\Gamma(2-\alpha)}{2} \frac{h^{1-\alpha}}{\Gamma(3-\alpha)}, \\
& \mathrm{B}=\left[(p-1)^{2-\alpha}-(p-2+\alpha) p^{1-\alpha}\right], \\
& \mathrm{C}^{\bar{a}}(j)=\left[(p-j+1)^{2-\alpha}-2(p-j)^{2-\alpha}+(p-j-1)^{2-\alpha}\right], \\
& \mathrm{C}^{\bar{b}}(j)=\left[(j+1)^{2-\alpha}-2 j^{2-\alpha}+(j-1)^{2-\alpha}\right], \\
& j=1, \ldots, p-1,
\end{aligned}
$$

and $(\cdot)^{\prime \prime}$ denotes second order derivative which is approximated utilising classical central finite difference scheme. Because of linearity assumption of the function $U$ on considered sub-intervals $\left[\xi_{i-1}, \xi_{i}\right]$ ([41]) the points $U_{0}^{\bar{a}}, U_{j}^{\bar{a}}, U_{j}^{\bar{b}}$ and $U_{p}^{\bar{b}}$ can be independent of discretization $\Delta \xi$. To understand how these points contribute the discretization points $\xi_{i}$, let us assume that point $\xi_{j}^{\bar{a}}$ falls between discretization points $\xi_{i-3}$ and $\xi_{i-2}$, we have

$$
U_{j}^{\bar{a}}=U_{i-3}\left(1-\frac{\xi_{j}^{\bar{a}}-\xi_{i-3}}{\Delta \xi}\right)+U_{i-2}\left(\frac{\xi_{j}^{\bar{a}}-\xi_{i-3}}{\Delta \xi}\right) .
$$

Therefore, in this example point $\xi_{j}^{\bar{a}}$ contributes the fractional stiffness matrix $\boldsymbol{K}$ in the row $i$ and the columns $i-3, i-2$ with coefficients $\left(1-\frac{\xi_{j}^{\bar{a}}-\xi_{i-3}}{\Delta \xi}\right)$ and $\left(\frac{\xi_{j}^{\bar{a}}-\xi_{i-3}}{\Delta \xi}\right)$, respectively. For $\alpha=1$ we recover the classical integer derivative of order 1 .

\subsection{Eringen non-local model}

The Eringen theory of non-local elasticity [15-17] is widely accepted and applied to many problems in the field of solid mechanics when scale effects must be taken into account. Eringen non-local theory assumes that the stress state at a reference point in the body is regarded to be dependent not only on the strain state at this point but also on the strain states at all other points of the body. This is in accordance with atomic theory of lattice dynamics and experimental observations on phonon dispersion.

Thus, the general constitutive equations can be written as

$$
\sigma_{i j}(\mathbf{x})=\int_{\Omega} \alpha\left(\left|\mathbf{x}^{\prime}-\mathbf{x}\right|, \mu\right) C_{i j k l} \varepsilon_{k l}\left(\mathbf{x}^{\prime}\right) d \Omega\left(\mathbf{x}^{\prime}\right),
$$

where $\sigma_{i j}(\mathbf{x})$ and $\varepsilon_{k l}(\mathbf{x})$ are, respectively, the components of the non-local stress tensor, and the infinitesimal strain tensor at point $\mathbf{x} . C_{i j k l}$ are the components of the fourth-order elastic tensor which depend of two constants for the case of isotropic materials.

The kernel function $\alpha\left(\left|\mathbf{x}^{\prime}-\mathbf{x}\right|, \mu\right)$ in Eq.(25) represents the non-local modulus. Euclidean Lagrangian distance is expressed by $\left|\mathbf{x}^{\prime}-\mathbf{x}\right|$ and the constant $\mu=$ $e_{0} a$ is a scale factor. Here $a$ is a characteristic length adjusted by the material constant $e_{0}$.

Among different admissible kernels which satisfy the normalization condition $\left(\int_{\Omega} \alpha\left(\left|\mathbf{x}^{\prime}-\mathbf{x}\right|, \mu\right) d \Omega\left(\mathbf{x}^{\prime}\right)=\mathbf{1}\right)$, the bell shaped Gaussian is widely used. According to [17], for this kind of attenuation kernel it is possible to represent the integral constitutive relations given by Eq.(25) in an equivalent differential form as

$$
\left(1-\left(e_{0} a\right)^{2} \nabla^{2}\right) \sigma_{i j}=C_{i j k l} \varepsilon_{k l},
$$

where $\nabla^{2}$ is the Laplacian operator.

Aydogdu [5] applied the above formulation to analyse the axial free vibration of an Eringen non-local elastic bar of constant cross section.

The problem is governed by the following equation [5]

$E \frac{\partial^{2} u}{\partial x^{2}}=\left(1-\ell_{g}^{2} \frac{\partial^{2}}{\partial x^{2}}\right) \rho \frac{\partial^{2} u}{\partial t^{2}}$,

where $\ell_{g}$ is the internal characteristic length. 
The temporal frequency for the non-local model, $\omega_{g}=\omega_{g}\left(\ell_{g}\right)$ is related to the corresponding to the local (classical) model $\omega_{L}\left(\ell_{g}=0\right)$ by:

$$
\frac{\omega_{g}}{\omega_{L}}=\frac{1}{\sqrt{1+\bar{\ell}_{g}^{2} \beta^{2}}} .
$$

For the above analysis it was assumed that $\lambda_{g}^{2} \bar{\ell}_{g}^{2}<1$, and $\beta$ and $\omega_{L}$ depend on the boundary condition imposed to the bar.

For the clamped-clamped case studied we have

$\beta=k \pi, \quad k \in \mathbb{Z}$,

and

$$
\omega_{L}=\frac{k \pi}{L} \sqrt{\frac{E}{\rho}} .
$$

\section{Analysis of natural frequencies and modal shapes}

In Sect. 2 we have introduced the non-dimensional notation. Summarizing, we have the following relations:

$$
\begin{aligned}
& \overline{\ell_{g}}=\frac{\ell_{g}}{L}, \\
& \overline{\ell_{f}}=\frac{2 \ell_{f}}{L}, \\
& \bar{\ell}=\frac{\overline{\ell_{g}}}{\overline{\ell_{f}}}=\frac{\ell_{g}}{2 \ell_{f}} .
\end{aligned}
$$

Now we additionally consider the following physical bounds:

$$
\left\{\begin{array} { l } 
{ \ell _ { g } \in [ 0 , L ] , } \\
{ \ell _ { f } \in ( 0 , \frac { L } { 2 } ] , }
\end{array} \Rightarrow \left\{\begin{array}{l}
\overline{\ell_{g}} \in[0,1], \\
\overline{\ell_{f}} \in(0,1] .
\end{array}\right.\right.
$$

It is worth to point out that $\alpha$ and $\ell_{f}$ may be considered as additional model parameters, like $\ell_{g}$ in Eringen model.

It should be emphasised that all results discussed below were obtained for constant $\Delta \xi=0.005$, keeping $h$ (Eq. (16)) of the same order. In this sense, the
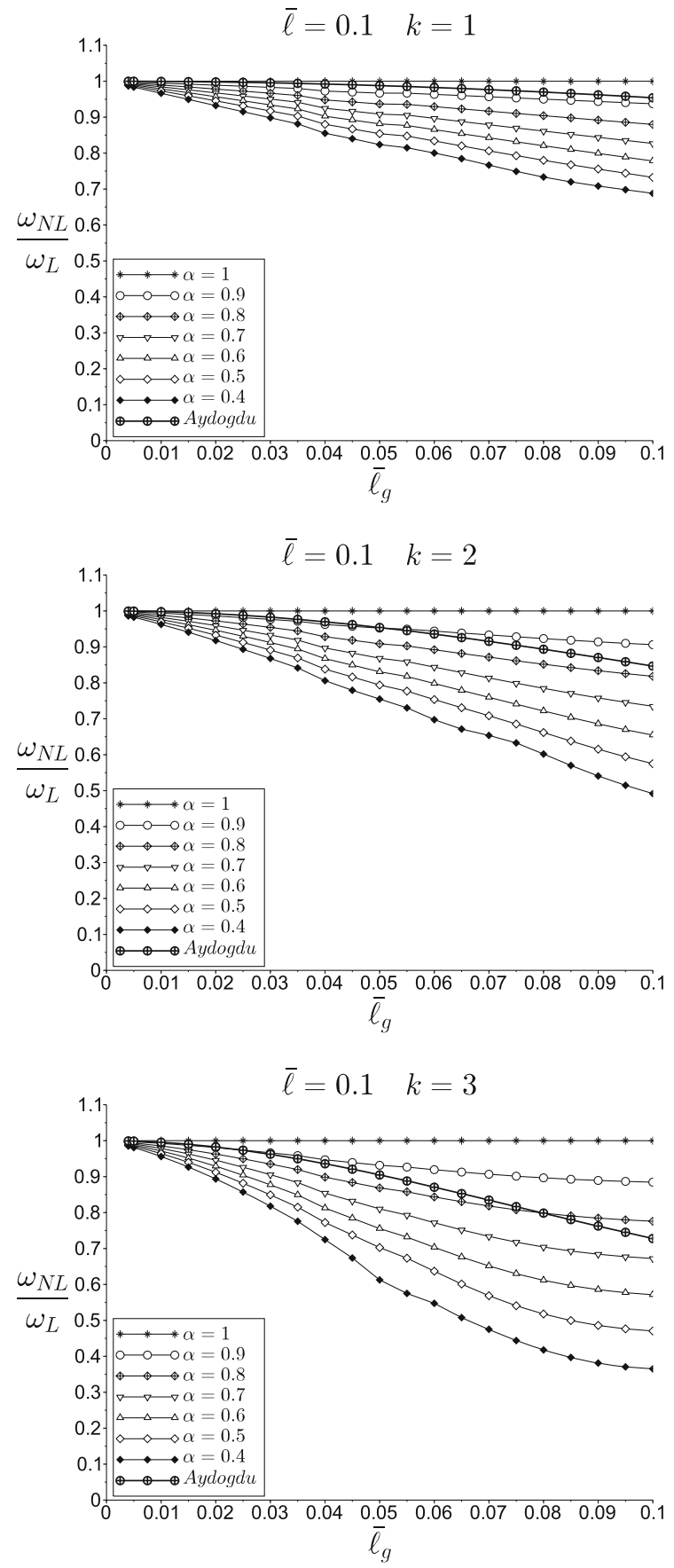

Fig. 3 The comparison of classical [5] versus fractional nonlocal models for small-scale effect on clamped-clamped nanorod for $\bar{\ell}=0.1$

parameter $p$ was changed in computations from $p=2$, when very small $\bar{\ell}_{f}$ was considered, up to $p=100$ when $\bar{\ell}_{f}$ had become comparable with the rod length. 

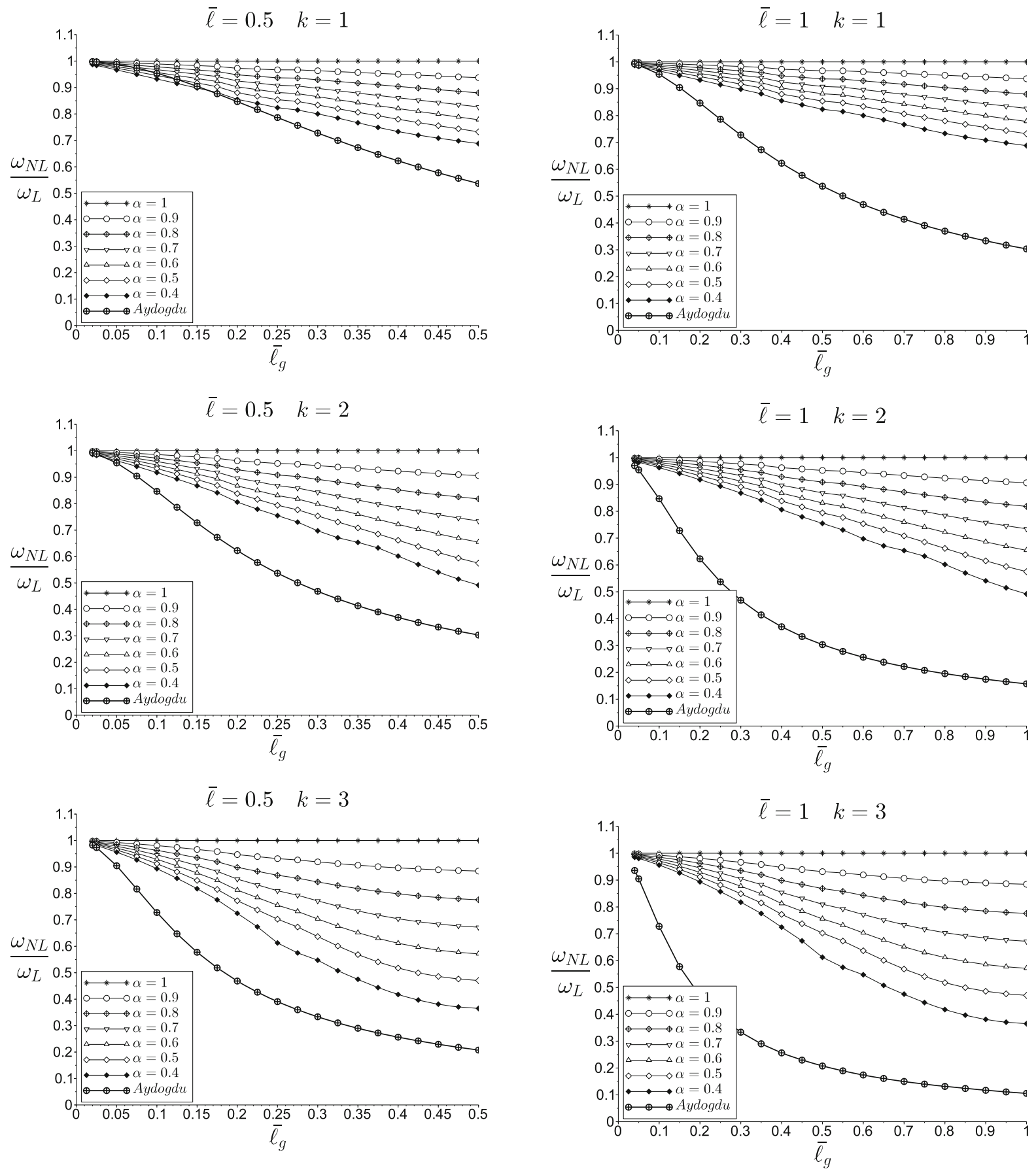

Fig. 4 The comparison of classical [5] versus fractional nonlocal models for small-scale effect on clamped-clamped nanorod for $\bar{\ell}=0.5$

In Figs. 3, 4, 5, 6, 7, 8, 9 the results contrasting the classical $(\alpha=1)$, Eringen [5] and fractional models are presented. Both natural frequencies and modal

Fig. 5 The comparison of classical [5] versus fractional nonlocal models for small-scale effect on clamped-clamped nanorod for $\bar{\ell}=1.0$

shapes are compared. Regarding natural frequencies, Figs. 3, 4, 5, 6 show the comparison of the first three natural frequencies $k \in\{1,2,3\}$, for different values 

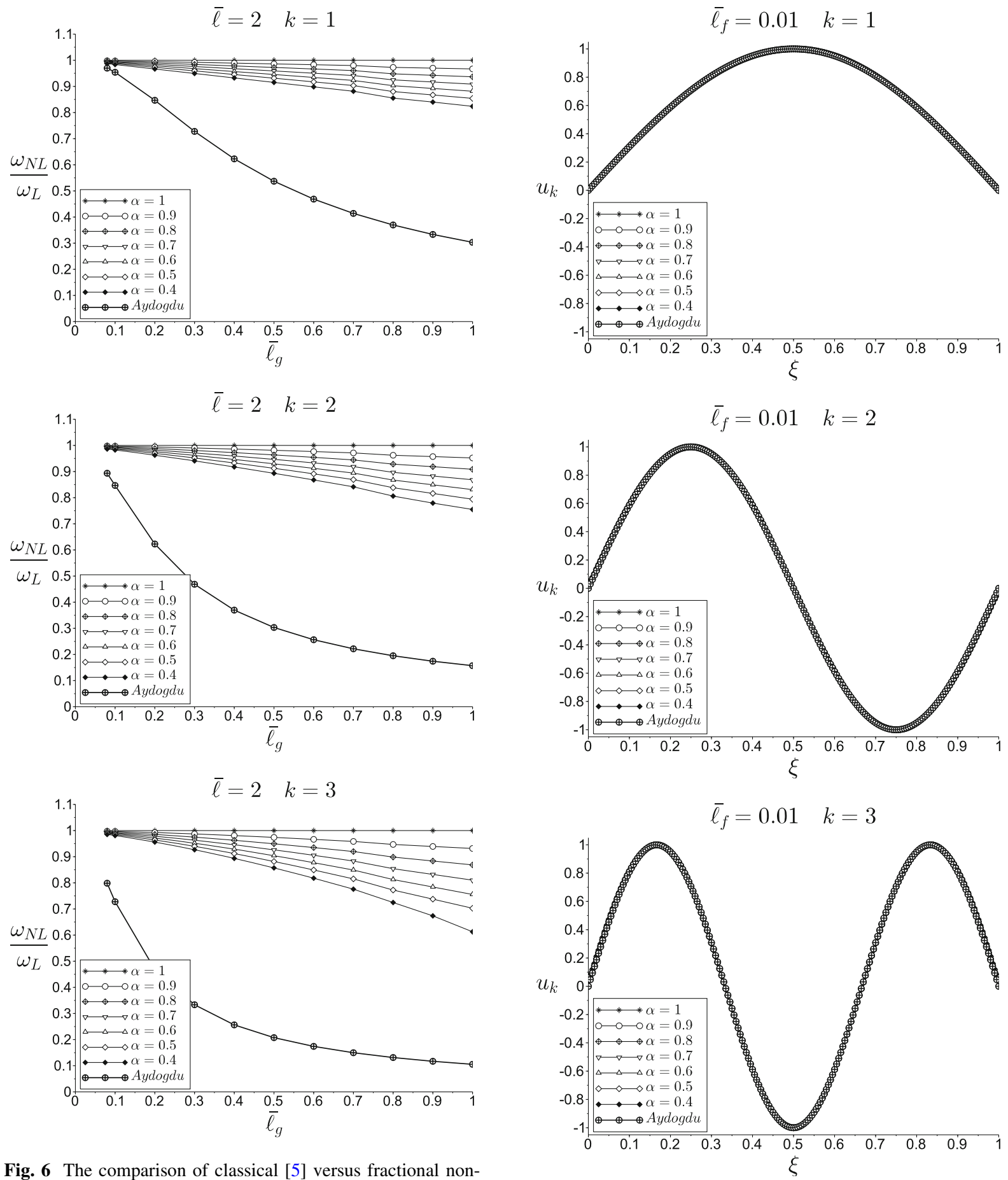

Fig. 6 The comparison of classical [5] versus fractional nonlocal models for small-scale effect on clamped-clamped nanorod for $\bar{\ell}=2.0$

of the Eringen and fractional length-scale parameters $\bar{\ell} \in\{0.1,0.5,1,2\}$, and also for different orders of fractiona continua $\alpha$. In these figures, $\omega_{L}$ represents the

Fig. 7 The comparison of classical [5] versus fractional nonlocal models-eigenmodes $k \in\{1,2,3\}$ and $\overline{\ell_{f}}=0.01$

frequency obtained with CCM and $\omega_{N L}$ the frequency obtained with the non-local model (either Eringen or FCM). We can observe that Eringen and fractional 

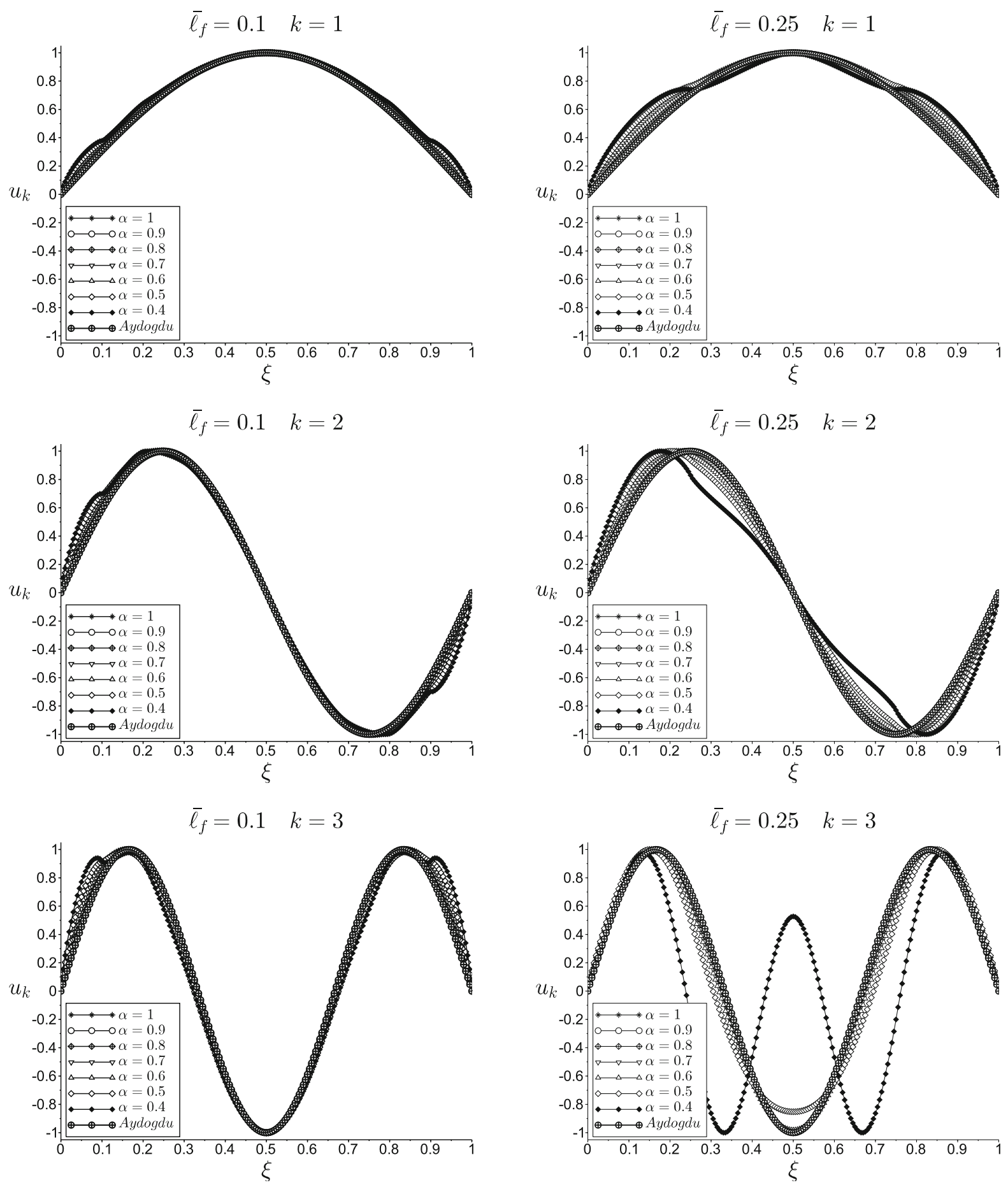

Fig. 8 The comparison of classical [5] versus fractional nonlocal models-eigenmodes $k \in\{1,2,3\}$ and $\overline{\ell_{f}}=0.1$

models converge with the local one when the values of the non-dimensional length-scale parameters $\ell_{g}$ and $\ell_{f}$ tend to zero, that is, when the length-scale parameters

Fig. 9 The comparison of classical [5] versus fractional nonlocal models-eigenmodes $k \in\{1,2,3\}$ and $\overline{\ell_{f}}=0.25$

of both models, $l_{g}$ and $l_{f}$, are small compared with the total length of the body $L$. Additionally, the results of the fractional model coincide with those of the 
classical one if the order of derivative is integer $(\alpha=1)$. Therefore, the length-scale parameter $\bar{\ell}_{f}$ does not play any role unless a fractional derivative order is considered.

The effect of increasing the length-scale parameters is similar in both non-local models, leading to a decrease in the natural frequencies which corresponds to a decrease in the stiffness of structure [5]. When the value of the length scales coincides in both formulations, the results are similar for a given value of the order of fractional continua $(\alpha=0.3)$. Since other values of $\alpha$ could be considered, the order of differentiation in FCM approach stands as an additional model parameter allowing a better calibration of the experimental results.

The first three modal shapes are depicted in Figs. 7 , 8,9 , for different values of the fractional length-scale parameter $\bar{\ell}_{f} \in\{0.01,0.1,0.25\}$ and for different orders of fractional continua $\alpha$. The modal shapes of the Eringen model are coincident with those obtained with CCM and therefore independent on the lengthscale parameter $\bar{\ell}_{g}$. Nevertheless, the modal shapes predicted by the fractional model show a dependence on $\bar{\ell}_{f}$ : at low values the non-local effects are negligible and the results of the FCM and CCM are consistent whereas large differences are observed for large values of $\bar{\ell}_{f}$. As with natural frequencies, FCM and $\mathrm{CCM}$ are also coincident for values of the derivative order $\alpha \approx 1$.

Recall that, in addition to $\alpha$ and $\ell_{f}$, one can also redefine the presented results using a different type of fractional derivative to obtain closer approximation of experimental observations.

\section{Conclusions}

This paper presents an analysis of the free axial vibrations of clamped nanorods using the FCM approach proposed by Sumelka $[80,78]$. The formulation uses the fractional Riesz-Caputo derivatives, which leads to two additional model parameters, $\alpha$ and $\ell_{f}$. This model is able to capture the size effect present at the nanoscale. The same problem has been solved by the classical nonlocal elasticity approach derived by Eringen [5, 17], which includes $\ell_{g}$ as length-scale parameter.
The natural frequencies and modal shapes are obtained with both procedures and the effects of the derivative order and length-scale parameters are compared and discussed. The following conclusions can be established:

- The natural frequencies obtained with both Eringen and fractional models converge with those derived from the local one when the length-scale parameter approaches zero, that is, when the length-scale is smaller in comparison to total length of the body.

- The results of the fractional model show that the length-scale parameter $\ell_{f}$ does not play any role unless a fractional derivative order is considered, and coincide with those of the classical one when the order of derivative is integer $(\alpha=1)$.

- The effect of increasing the length-scale parameters is similar in both non-local models, leading to a decrease in the natural frequencies. When equal length scales are used in both formulations, the results approximately coincide when the order of fractional continua is around $\alpha=0.3$. It should be stated that the order of differentiation in FCM approach stands as an additional model parameter allowing a better calibration of the experimental results.

- Finally, it is worth to note that while the modal shapes calculated with the Eringen model are independent on the length-scale parameter $\ell_{g}$, and coincident with those obtained with Classical Continuum Mechanics, the modal shapes predicted by the fractional model show a dependence on $\ell_{f}$ and $\alpha$.

Both theories include non-local parameters that must be selected for each specific material based on experimental results, molecular dynamics analysis or ab initio formulations. Since the proposed fractional approach includes two non-local parameters, it is more suitable for predicting the observed behavior in a given application. It is worth to point out that it is also possible to redefine the presented analysis using a different type of fractional derivative.

Open Access This article is distributed under the terms of the Creative Commons Attribution License which permits any use, distribution, and reproduction in any medium, provided the original author(s) and the source are credited. 


\section{References}

1. Agrawal OP (2007) Fractional variational calculus in terms of Riesz fractional derivatives. J Phys A 40(24):6287-6303

2. Ansari R, Shahabodini A, Rouhi H (2013) A thickness-independent nonlocal shell model for describing the stability behavior of carbon nanotubes under compression. Compos Struct 100:323-331

3. Aranda-Ruiz J, Loya J, Fernández-Sáez J (2012) Bending vibrations of rotating nonuniform nanocantilevers using the eringen nonlocal elasticity theory. Compos Struct 95:2990-3001

4. Atanackovic TM, Stankovic B (2009) Generalized wave equation in nonlocal elasticity. Acta Mech 208(1-2):1-10

5. Aydogdu M (2009) Axial vibration of the nanorods with the nonlocal continuum rod model. Physica E 41:861-864

6. Bourlon B, Glattli DC, Miko C, Forro L, Bachtold A (2004) Carbon nanotube based bearing for rotational motions. Nano Lett 4:709-712

7. Carpinteri A, Cornetti P, Sapora A (2011) A fractional calculus approach to nonlocal elasticity. Eur Phys J Spec Top 193:193-204

8. Carpinteri A, Cornetti P, Sapora A (2014) Nonlocal elasticity: an approach based on fractional calculus. Meccanica 49:2551-2569

9. Carpinteri A, Cornetti P, Sapora A, Di Paola M, Zingales M (2009) Fractional calculus in solid mechanics: local versus non-local approach. Phys Scr T136:7

10. Chen Y, Lee JD, Eskandarian A (2004) Atomistic viewpoint of the applicability of microcontinuum theories. Int J Solids Struct 41(8):2085-2097

11. Cottone G, Di Paola M, Zingales M (2009) Elastic waves propagation in $1 \mathrm{D}$ fractional non-local continuum. Physica E 42:95-103

12. Di Paola M, Zingales M (2008) Long-range cohesive interactions of non-local continuum faced by fractional calculus. Int J Solids Struct 45:5642-5659

13. Drapaca CS, Sivaloganathan S (2012) A fractional model of continuum mechanics. J Elast 107:107-123

14. Drexler KE (ed) (1992) Nanosystems: molecular machinery, manufacturing, and computation. Wiley, New York

15. Eringen AC (1972) Linear theory of nonlocal elasticity and dispersion of plane-waves. Int J Eng Sci 10(5):233-248

16. Eringen AC (1972) Nonlocal polar elastic continua. Int J Eng Sci 10:1-16

17. Eringen AC (1983) On differential-equations of nonlocal elasticity and solutions of screw dislocation and surfacewaves. J Appl Phys 54(9):4703-4710

18. Eringen AC, Edelen DGB (1972) Nonlocal elasticity. Int J Eng Sci 10(3):233-248

19. Fakhrabadi MMS, Rastgoo A, Ahmadian MT (2014) Size-dependent instability of carbon nanotubes under electrostatic actuation using nonlocal elasticity. Int J Mech Sci 80:144-152

20. Fennimore A, Yuzvinsky TD, Han WQ, Fuhrer MS, Cumings J, Zettl A (2003) Rotational actuators based on carbon nanotubes. Nature 424:408

21. Firouz-Abadi RD, Fotouhi MM, Haddadpour H (2011) Free vibration analysis of nanocones using a nonlocal continuum model. Phys Lett A 375:3593-3598

22. Fleck NA, Hutchinson JW (1997) Strain gradient plasticity. Adv Appl Mech 33:295-361
23. Frederico GSF, Torres DFM (2010) Fractional Noether's theorem in the Riesz-Caputo sense. Appl Math Comput 217:1023-1033

24. Ghavanloo E, Fazelzadeh SA (2013) Nonlocal elasticity theory for radial vibration of nanoscale spherical shells. Eur J Mech Solids. doi:10.1016/j.euromechsol.2013.02.003

25. Ghavanloo E, Fazelzadeh SA (2013) Radial vibration of free anisotropic nanoparticles based on nonlocal continuum mechanics. Nanotechnology 24:075702

26. Green AE, Rivlin RS (1964) Multipolar continuum mechanics. Arch Rational Mech Anal 17(2):113-147

27. Han J, Globus A, Jaffe R, Deardorff G (1997) Molecular dynamics simulation of carbon nanotubebased gear. Nanotechnology 8:95-102

28. Heireche H, Tounsi A, Benzair A, Maachou M, Adda Berdia EA (2008) Sound wave propagation in single-walled carbon nanotubes using nonlocal elasticity. Physica E 40:2791-2799

29. Herbold EB, Nesterenko VF (2007) Solitary and shock waves in discrete strongly nonlinear double power-law materials. Appl Phys Lett 90:261902

30. Hosseini-Hashemi Shahrokh, Zare Mojtaba, Nazemnezhad Reza (2013) An exact analytical approach for free vibration of mindlin rectangular nano-plates via nonlocal elasticity. Compos Struct 100:290-299

31. Hua YG, Liew KM, Wang Q, He XQ, Yakobson BI (2008) Nonlocal shell model for elastic wave propagation in singleand double-walled carbon nanotubes. J Mech Phys Solids 56:3475-3485

32. Ke LL, Wang YS, Wang ZD (2012) Nonlinear vibration of the piezoelectric nanobeams based on the nonlocal theory. Compos Struct 94(6):2038-2047

33. Ke Liao-Liang, Wang Yue-Sheng, Wang Zheng-Dao (2008) Non-local elastic plate theories. Proc R Soc A 463:3225-3240

34. Kiani K (2010) Free longitudinal vibration of tapered nanowires in the context of nonlocal continuum theory via a perturbation technique. Physica E 43(1):387-397

35. Kilbas AA, Srivastava HM, Trujillo JJ (2006) Theory and applications of fractional differential equations. Elsevier, Amsterdam

36. Klimek M (2001) Fractional sequential mechanics-models with symmetric fractional derivative. Czechoslov J Phys 51(12):1348-1354

37. Kröner E (1963) On the physical reality of torque stresses in continuum mechanics. Int J Eng Sci 1:261-278

38. Kroner E (1967) Elasticity theory of materials with longrange cohesive forces. Int J Solids Struct 3:731-742

39. Lam DCC, Yang F, Chong ACM, Wang J, Tong P (2003) Experiments and theory in strain gradient elasticity. J Mech Phys Solids 51:1477-1508

40. Lazopoulos KA (2006) Non-local continuum mechanics and fractional calculus. Mech Res Commun 33:753-757

41. Leszczyński JS (2011) An introduction to fractional mechanics. Monographs No 198. The Publishing Office of Czestochowa University of Technology, Czestochowa

42. Li Ch (2014) Torsional vibration of carbon nanotubes: comparison of two nonlocal models and a semi-continum model. Int J Mech Sci 82:25-31

43. Liew KM, Wei JX, He XQ (2007) Carbon nanocones under compression: buckling and post-buckling behaviors. Phys Rev B Condens Matter Mater Phys 75:195435 
44. Loya J, Lopez-Puente J, Zaera R, Fernandez-Saez J (2009) Free transverse vibrations of cracked nanobeams using a nonlocal elasticity model. J Appl Phys 105(4):044309

45. Loya JA, Aranda-Ruiz J, Fernández-Sáez J (2014) Torsion of cracked nanorods using a nonlocal elasticity model. J Phys D Appl Phys 47:115304

46. Lu P (2007) Dynamic analysis of axially prestressed micro/nanobeam structures based on nonlocal beam theory. J Appl Phys 101(7):073504

47. Mainardi F (2010) Fractional calculus and waves in linear viscoelasticity. Imperial College Press, London

48. Maranganti R, Sharma P (2007) A novel atomistic approach to determine strain-gradient elasticity constants: tabulation and comparison for various metals, semiconductors, silica, polymers and the (ir) relevance for nanotechnologies. J Mech Phys Solids 55:1823-1852

49. Marsden JE, Hughes TJH (1983) Math Found Elast. Prentice-Hall, New Jersey

50. Martin CR (1996) Membrane-based synthesis of nanomaterials. Chem Mater 8:1739-1746

51. Mindlin RD (1964) Micro-structure in linear elasticity. Arch Rationa Mech Anal 16(1):51-78

52. Mindlin RD (1965) Second gradient of strain and surfacetension in linear elasticity. Int J Solids Struct 1(4):417-438

53. Mindlin RD, Eshel N (1968) On first strain-gradient theories in linear elasticity. Int J Solids Struct 4(1):109-124

54. Mindlin RD, Tiersten HF (1962) Effects of couple-stresses in linear elasticity. Arch Ration Mech Anal 11(5):415-448

55. Moosavi H, Mohammadi M, Farajpour A, Shahidi SH (2011) Vibration analysis of nanorings using nonlocal continuum mechanics and shear deformable ring theory. Physica E 44:135-140

56. Murmu T, Adhikari S (2010) Non local effects in the longitudinal vibration of double-nanorod systems. Physica E 43(1):415-422

57. Murmu T, Adhikari S (2010) Scale-dependent vibration analysis of prestressed carbon nanotubes undergoing rotation. J Appl Phys 108:123507

58. Murmu T, Pradhan SC (2009) Small-scale effect on the free in-plane vibration of nanoplates by nonlocal continuum model. Physica E 41(8):1628-1633

59. Murmu T, Pradhan SC (2009) Small-scale effect on the vibration of nonuniform nanocantilever based on nonlocal elasticity theory. Physica E 41(8):1451-1456

60. Murmu T, Pradhan SC (2009) Thermo-mechanical vibration of a single-walled carbon nanotube embedded in an elastic medium based on nonlocal elasticity theory. Comput Mater Sci 46(4):854-859

61. Narendar S (2011) Terahertz wave propagation in uniform nanorods: a nonlocal continuum mechanics formulation including the effect of lateral inertia. Physica $E$ 43(4):1015-1020

62. Narendar S, Gopalakrishnan S (2010) Ultrasonic wave characteristics of nanorods via nonlocal strain gradient models. J Appl Phys 107(8):084312

63. Narendar S, Gopalakrishnan S (2011) Critical buckling temperature of single-walled carbon nanotubes embedded in a one-parameter elastic medium based on nonlocal continuum mechanics. Physica E 43(6):1185-1191

64. Narendar S, Gopalakrishnan S (2011) Nonlocal wave propagation in rotating nanotube. Physics 1:17-25
65. Odibat Z (2006) Approximations of fractional integrals and Caputo fractional derivatives. Appl Math Comput 178:527-533

66. Park SK, Gao XL (2008) Variational formulation of a modified couple stress theory and its application to a simple shear problem. Zeitschrift fur Angewandte Mathematik und Physik 59:904-917

67. Park SK, Gao X-L (2008) Variational formulation of a modified couple stress theory and its application to a simple shear problem. Zeitschrift fur angewandte Mathematik und Physik 59:904-917

68. Peddieson J, Buchanan GR, McNitt RP (2003) Application of nonlocal continuum models to nanotechnology. Int J Eng Sci 41(3-5):305-312

69. Podlubny I (1999) Fractional differential equations, volume 198 of mathematics in science and engineering. Academic Press, London

70. Pradhan SC, Murmu T (2010) Application of nonlocal elasticity and DQM in the flapwise bending vibration of a rotating nanocantilever. Physica E 42:1944-1949

71. Reddy JN (2007) Nonlocal theories for bending, buckling and vibration of beams. Int J Eng Sci 45(2-8):288-307

72. Saji VS, Choe HC, Young KWK (2010) Nanotechnology in biomedical applications-a review. Int $\mathrm{J}$ Nano Biomater 3:119-139

73. Sapora A, Cornetti P, Carpinteri A (2013) Wave propagation in nonlocal elastic continua modelled by a fractional calculus approach. Commun Nonlinear Sci Numer Simul 18:63-74

74. Seifoori S, Liaghat GH (2013) Low velocity impact of a nanoparticle on nanobeams by using a nonlocal elasticity model and explicit finite element modeling. Int J Mech Sci 69:85-93

75. Sudak LJ (2003) Column buckling of multiwalled carbon nanotubes using nonlocal continuum mechanics. J Appl Phys 94(11):7281-7287

76. Sumelka W (2013) Fractional deformation gradients. In: 7th International workshop on dynamic behaviour of materials and its applications in industrial processes, Madrid, Spain, pp 54-55

77. Sumelka W (2013) Non-local continuum mechanics based on fractional calculus. In: 20th International Conference on Computer Methods in Mechanics, Poznań, Poland, 27-31 Aug 2013, pp MS02-05-06

78. Sumelka W (2014) Application of fractional continuum mechanics to rate independent plasticity. Acta Mech. doi:10.1007/s00707-014-1106-4

79. Sumelka W (2014) Fractional viscoplasticity. Mech Res Commun 56:31-36

80. Sumelka W (2014) Thermoelasticity in the framework of the fractional continuum mechanics. J Therm Stress 37(6):678-706

81. Sun CT, Zhang HT (2003) Size-dependent elastic moduli of platelike nanomaterials. J Appl Phys 93(2):1212-1218

82. Tarasov VE (2006) Continuous limit of discrete systems with long-range interaction. J Phys A Gen Phys 39:14895-14910

83. Tarasov VE (2013) Lattice model with power-law spatial dispersion for fractional elasticity. Cent Eur J Phys 11:1580-1588

84. Toupin RA (1963) Elastic materials with couple-stresses. Arch Ration Mech Anal 11(5):385-414

85. Toupin RA (1964) Theories of elasticity with couple-stress. Arch Ration Mech Anal 17(2):85-112 
86. Tsai P, Fang T (2007) A molecular dynamics study of the nucleation, thermal stability and nanomechanics of carbon nanocones. Nanotechnology 18:105702

87. Vila J, Zaera R, Fernández-Sáez J (2015) Axisymmetric free vibration of closed thin spherical nanoshell with bending effects. J Vib Control. doi:10.1177/1077546314565808

88. Wang CM, Duan WH (2008) Vibration analysis of nanorings using nonlocal continuum mechanics and shear deformable ring theory. J Appl Phys 104:014303

89. Wang CM, Zhang YY, He XQ (2007) Vibration of nonlocal timoshenko beams. Nanotechnology 18(10):105401

90. Wang CM, Zhang YY, Ramesh SS, Kitipornchai S (2006) Buckling analysis of micro- and nano-rods/tubes based on nonlocal timoshenko beam theory. J Phys D Appl Phys 39(17):3904-3909

91. Wang Q, Varadan VK (2007) Application of nonlocal elastic shell theory in wave propagation analysis of carbon nanotubes. Smart Mater Struct 16:178-190
92. Wang Q, Wang CM (2007) The constitutive relation and small scale parameter of nonlocal continuum mechanics for modelling carbon nanotubes. Nanotechnology 18:075702

93. Xu MT (2006) Free transverse vibrations of nano-to-micron scale beams. Proce R Soc Math Phys Eng Sci 462(2074):2977-2995

94. Yang F, Chong ACM, Lam DCC, Tong P (2002) Couple stress based strain gradient theory for elasticity. Int J Solids Struct 39:2731-2743

95. Yang JFC, Lakes RS (1982) Experimental-study of micropolar and couple stress elasticity in compact-bone in bending. J Biomech 15:91-98

96. Zaera R, Fernandez-Saez J, Loya JA (2013) Axisymmetric free vibration of closed thin spherical nano-shell. Compos Struct 104:154-161

97. Zhou SJ, Li ZQ (2001) Length scales in the static and dynamic torsion of a circular cylindrical micro-bar. J Shandong Univ Technol 31(5):401-407 